\title{
EXTRAÇÃO DE TANINOS DE FARINHA DE SEMENTE DE GRAVIOLA POR ULTRASSOM
}

Extraction of graviola seed flour tanks by ultrassom

Extracción de taninos de harina de semilla de graviola por ultrasom

V. M. Alves*1, K. A. Amorim², D. J. M. Abreu², J. S. Silva³, E. R. Asquieri $^{4}$, C. Damiani ${ }^{4}$

${ }^{1}$ Departamento de Ciências dos Alimentos - Universidade Federal Tocantins,

${ }^{2}$ Departamento de Engenharia de Alimentos - Universidade Federal de Goiás, Goiânia, GO, Brasil.

${ }^{3}$ Departamento de Ciência dos Alimentos - Universidade Federal de Lavras, Lavras, MG, Brasil.

${ }^{4}$ Departamento de Engenharia de Alimentos - Universidade Federal de Goiás, Goiânia, GO, Brasil.

*Correspondência: Departamento de Ciências dos Alimentos - Universidade Federal Tocantins, Instituto de Ciência e Tecnologia de Alimentos - CEP: 77001-090-Palmas, TO, Brasil, Telefone: (63) 3229-4305, e-mail: vanialvees@gmail.com

\section{RESUMO}

Os taninos são compostos fenólicos, responsáveis pela adstringência de muitos frutos e produtos vegetais, devido à precipitação de glicoproteínas salivares, o que ocasiona a perda do poder lubrificante, sendo considerado antinutricional. Com isso, o objetivo desse estudo foi avaliar a extração de taninos totais da farinha de semente de graviola, utilizando banho ultrassônico com diferentes solventes. Os taninos foram extraídos, por meio de banho ultrassônico, por 30 minutos, a temperatura ambiente, utilizando como solventes o etanol 70\%, água destilada e metanol 50\%. Os taninos totais foram determinados, de acordo com a metodologia de Folin-Denis. Observou-se uma extração de 572,93, 589,76, 689,46 (mg equivalente de ácido tânico/100g de amostra), utilizando água destilada, etanol 70\% e metanol 50\% respectivamente. Não observou-se diferença significativa entre os solventes, além disso, foi possível observar que a quantidade de taninos encontrada na farinha de semente de graviola não é nociva à saúde, podendo esta ser implementada na alimentação humana.

Palavras-chave: compostos fenólicos, adstringência, Annona muricata L.

\section{ABSTRACT}

Tannins are phenolic derivatives, responsible for the addition of many fruits and vegetable products, due to the precipitation of salivary glycoproteins, which causes the loss of lubricating power, considered antinutritional. This study was applied a therapy of extraction of different levels of vitamin from soursop for ultrasonic bath with different solvents. The tannins were extracted through the ultrasonic bath for 30 minutes at room temperature while solvents in 70\% ethanol, distilled water and 50\% methanol, the tannins were extracted according to the Folin-Denis methodology. An extraction of 572.93, 589.76, 689.46 (equivalent to tannic acid / 100g of sample) distilled water, $70 \%$ ethanol and 50\% methanol respectively, was not observed significant difference between the solvents, in addition The weight dose can not be compared to health, but can be applied to human food.

Keywords: phenolic compounds, astringency, Annona muricata L.

\section{RESUMEN}

Los taninos son compostos fenólicos, responsables de la comunicación de los frutos y los frutos vegetales, los resultados de la producción de glicoproteínas, y la ocasión para compartir el poder y el antinutricional. Com isso, 
o target desse isudo foi avaliar a extração of taninos totais da farinha de semente de graviola, utilizando banho ultrassônico com diferentes solventes. Os taninos foram extraídos, por meio de banho ultrassônico, por 30 minutos, a temperatura ambiente, utilizando como solventes etanol al 70\%, água destilada y metanol al 50\%. Os taninos totais foram por acordo com a metodologia de Folin-Denis. Observación adicional de 572,93, 589,76, 689,46 (mg equivalente de ácido tânico / $100 \mathrm{~g}$ de amostra), utilizando água destilada, etanol $70 \%$ y metanol 50\% respectivamente. No hay diferencias significativas en los solventes, también se puede observar la cantidad de taninos que se encuentran en la farina de la gravedad de la vida, pero que no se han implementado, sino que se ha implementado en la alimentación.

Palabras clave: compuestos fenólicos, astringencia, Annona muricata L.

\section{INTRODUÇÃO}

Nos últimos anos, a população em geral tem dedicado mais atenção para o papel da dieta na saúde humana. Vários estudos epidemiológicos indicam que o consumo frequente de frutas e vegetais está associado com baixa incidência de doenças crônicas, doenças cardiovasculares, inflamações, artrites e câncer, resultado particularmente atribuído aos compostos com atividade antioxidante (OLIVEIRA et al., 2011).

Os efeitos protetores das frutas e vegetais têm sido atribuídos à presença de diversas substâncias em suas composições, sendo os principais a vitamina $C$, carotenoides e os mais frequentemente encontrados, compostos fenólicos (SILVA et al., 2010; OLIVEIRA et al., 2011).

Nos alimentos, estes compostos podem influenciar o valor nutricional e a qualidade sensorial, conferindo atributos como cor, textura, amargor e adstringência (EVERETTE et al., 2010).

Em função da elevada atividade antioxidante que possuem, uma gama de compostos fenólicos desempenha papel importante nos processos de inibição do risco das doenças cardiovasculares e podem atuar sobre o estresse oxidativo, relacionado com diversas patologias crônico-degenerativas, como o diabetes, o câncer e processos inflamatórios (ROCHA et al., 2011). Entretanto, quando em concentração muito elevada ou em composição inadequada, estes compostos podem conferir características indesejáveis, como o escurecimento enzimático de frutas e a interação com proteínas, carboidratos e minerais (IMEH e KHOKHAR, 2002).

Os compostos fenólicos possuem estruturas, desde as mais simples como os ácidos fenólicos até os polímeros mais complexos com alto peso molecular como os taninos, que são encontrados em muitas frutas, sendo caracterizados como compostos fenólicos de alto peso molecular, entre 500 e 3000 Dalton (SARKAR e HOWARTH, 1976).

Os taninos, em pequenas quantidades em frutos, conferem-lhes características sensoriais desejáveis, no entanto, quantidades maiores conferem aos frutos e outros alimentos características adstringentes. Os taninos possuem a capacidade de formar complexos insolúveis em água com proteína, por se ligarem covalentemente, além de interagirem com minerais divalentes, como ferro não-heme e zinco, diminuindo a absorção do mesmo no lúmen intestinal (Silva e Silva, 1999). Sendo assim, os taninos são considerados antinutrionais (SALUNKE et al., 1990).

O Brasil tem se sobressaído como um respeitável produtor e consumidor de frutas, e a Graviola (Annona muricata L.), conhecida também como guanabara, araticum, coração-de-rainha, frutadoconde, jaca-do-pará, pinha, entre outros, pertence à família Annonaceae e ao gênero Annona. Os frutos são do tipo baga, com polpa mucilaginosa e levemente ácida. Originária da América tropical, principalmente das Antilhas e da América Central, é muito cultivada nos países de clima tropical, inclusive no Brasil 
(VIEIRA, 2010; CORRÊA, 1984). O fruto é empregado, principalmente, na indústria de polpas alimentícias para refrescos, geleias, doces e sorvetes (NUNES et al., 2013). Durante o processamento de frutos, vários resíduos são gerados, cerca de $40 \%$ da produção, compostos por restos de polpa, talos, casca e sementes (HENRIQUE et al., 2013), onde se encontra concentrações consideráveis de diferentes compostos, como pectinas, lipídios, flavonoides, fibras dietéticas e compostos bioativos, tendo esses um potencial, se recuperado de maneira correta e eficiente.

Sendo assim, a extração é um passo importante para o isolamento, identificação e utilização destes compostos, considerando a grande diversidade química de compostos fenólicos distribuídos na natureza, diferentes solventes são empregados no processo de extração e diferentes metodologias analíticas são empregadas no processo de quantificação destes compostos (EFRAIN et al., 2006).

O sistema ultrassom oferece uma série de vantagens em termos de rendimento, produtividade e seletividade, além de redução do uso de solventes químicos tornando-se um dos principais "eco friendlyprocess" (CHEMART e KHAN, 2011). Assim sendo, o objetivo desse estudo foi avaliar a extração de taninos totais de farinha de semente de graviola, utilizando banho ultrassônico com diferentes solventes.

\section{MATERIAL E MÉTODOS}

As sementes de graviola foram adquiridas, por meio de doação da empresa Frutos do Brasil, com sede em Goiânia - Goiás, cujas sementes eram da safra 2016/2017. Após a separação das polpas e sementes do fruto de graviola, as sementes foram acondicionadas em sacos plásticos de polietileno de baixa densidade e transportadas, imediatamente, ao
Laboratório de Tecnologia de Produtos de Origem Vegetal, do Setor de Engenharia de Alimentos, da Escola de Agronomia, da Universidade Federal de Goiás (EA/UFG).

Ao chegar no laboratório, as sementes de graviola foram lavadas e sanitizadas em solução de hipoclorito de sódio $(\mathrm{NaOCl})$, a $200 \mathrm{ppm}$ por 30 minutos, com a finalidade de eliminar todas as formas vegetativas de microrganismos e evitar deterioração pelo tempo de armazenamento. Em seguida, as sementes foram secas em centrifuga especial para vegetais e armazenadas em novos sacos de polietileno de baixa densidade e acondicionadas em freezer à $18^{\circ} \mathrm{C}$ até o processamento da farinha. As sementes foram processadas em liquidificador para a obtenção da farinha.

Os taninos foram extraídos da seguinte maneira: pesou-se, em diferentes béqueres, um grama de amostra seca à $50^{\circ} \mathrm{C}$ por 21 horas em estufa de circulação de ar forçado. Em seguida, adicionou-se, em cada béquer, $10 \mathrm{~mL}$ de cada solvente, sendo eles etanol 70\% (ET70), água destilada (AD) e metanol $50 \%$ (MET50). Posteriormente, as amostras foram submetidas ao banho ultrassônico à $60 \mathrm{hz} / \mathrm{Krz}$ por 30 minutos a temperatura ambiente. Logo após este processo, os extratos foram filtrados, com papel filtro e armazenados em fraco âmbar, para posterior determinação dos taninos totais. As extrações foram realizadas com três repetições (FAN et al., 2018).

A determinação dos taninos totais foi realizada de acordo com a metodologia de FolinDenis (AOAC, 1980). O ensaio seguiu utilizando 0,1 $\mathrm{mL}$ do extrato obtido anteriormente, $8,4 \mathrm{~mL}$ de água destilada e $0,5 \mathrm{~mL}$ de do reagente Folin-Denis, além de 1,0 mL de Carbonato de sódio saturado. Os tubos foram agitados e deixados em repouso por 30 minutos ao abrigo da luz. A leitura foi feita em 
espectrofotômetro (Rayleygh UV-1800) à $760 \mathrm{~nm}$. Todos os extratos foram lidos em triplicata e os resultados expressos em mg equivalente de ácido tânico/100g.

O experimento foi montado em delineamento inteiramente casualizado (DIC) com 3 tratamento, sendo: ET70: Etanol 70\%; MET50: Metanol 50\%; AD: Água destiladae 3 repetições.

Os dados foram analisados por teste de Tukey $(\mathrm{p}<0,05)$ para avaliar a diferença significativa entre os tratamentos. Os resultados foram expressos em média \pm desvio padrão. A análise estatística foi realizada no software SISVAR 5.6 (FERREIRA, 2011).

\section{RESULTADOS E DISCUSSÃO}

Os resultados do conteúdo de taninos totais extraídos da farinha de sementes de graviola pelo banho ultrassom com diferentes solventes podem ser observados na Tabela 1.

Tabela 1. Conteúdo de taninos totais da farinha de semente de graviola extraídos com diferentes solventes em banho ultrassônico.

\begin{tabular}{cc}
\hline Solventes* & $\begin{array}{c}\text { Taninos totais } \\
\end{array}$ \\
\hline AD & $572,93^{\mathrm{a}}$ \\
ET70 & $589,76^{\mathrm{a}}$ \\
MET50 & $686,46^{\mathrm{a}}$ \\
\hline
\end{tabular}

*ET70: Etanol 70\%; MET50: Metanol 50\%; AD: Água destilada;

**Letras diferentes na mesma coluna diferem entre si pelo teste de Tukey a 5\% de significância.

De acordo com a tabela 1, observou-se que não houve diferença significativa $(\mathrm{p}<0,05)$ entre os diferentes solventes, sendo assim, qualquer um dos solventes pode ser utilizado para extração de taninos totais, porém a água seria o mais recomendado por não agredir o meio ambiente e, também, pelo fato de que a maioria dos compostos fenólicos, como os taninos, não é encontrada no estado livre na natureza, mas sob forma de ésteres ou de heterosídeos sendo, portanto, solúveis em água e em solventes orgânicos polares (MONTEIRO et al., 2005).

A ingestão excessiva de taninos podem causar efeitos adverso e, em muitos casos, serem considerados antinutricionais, porque precipitam proteínas, inibem enzimas digestivas e afetam a utilização de vitaminas e minerais podendo, ainda, em alta concentração, desenvolver câncer de bochecha e esôfago (MONTEIRO et al., 2005).

Um estudo avaliou o efeito dos taninos em modelos animais e concluiu que doses $0,5 \mathrm{a} 2 \mathrm{~g} / \mathrm{kg}$ / dia (5\% da dieta) não apresentou toxidade aguda, porém afetou o crescimento e que concentrações mais baixas não causaram nenhum efeito negativo. Sendo assim, a farinha da semente de graviola pode ser utilizada na alimentação humana, pois a quantiade de taninos totais contida na farinha é pequena, não sendo nociva a saúde (MENNEN et al., 2005).

\section{CONCLUSÃO}

Os extratos obtidos da farinha da semente de graviola demonstraram considerável concentração de taninos totais, podendo ser extraido utilizando 
qualquer um dos três solventes avaliados, ou seja, agua, etanol ou metanol. A farinha de graviola pode ser utilizada na alimentação humana de forma segura, por não apresentar quantidades suficientes de

\section{RERERÊNCIAS}

ASSOCIATION OF OFFICIAL ANALYTICAL CHEMISTRY. Official methods of analysis. Washington, D. C., 13thed, 1018p, 1980.

CHEMART, F. e KHAN, M. K. Applications of ultrasound in food technology: Processing, preservation and extraction. Ultrasonics Sonochemistry, v. 18, p. 813-835, 2011.

EFRAIM, P.; TUCCI, M. L.; PEZOA-GÂRCIA, N. H.; HADAD, R.; EBERLIN, M. N. Teores de compostos fenólicos de sementes de cacaueiro de diferentes genótipos. Brazilian Journal of Food Technology, Campinas, v. 9, n. 4, p. 229-236, 2006.

ESCRIVA, C.; VIANA, E.; MOLTO, J. C.; PICO, Y.; MANES, J. Comparison of four methods for the determination of polycyclic aromatic hydrocarbons in airborne particulates. Journal of Chromatography, v. 676, n. 2, p.375-388, 1994.

EVERETTE, J. D.; BRYANT, Q. M.; GREEN, A. M.; ABBEY, Y. A.; WANGILA, G. W.;WALKER, R. B. Thorough study of reactivity of various compound classes toward the Folin-Ciocalteou reagent. Journal of Agricultural and Food Chemistry, Washington, v. 58, p. 8.139-8.144, 2010.

FAN, H.; SUN, L.; YANG, L.; ZHOU, J.; YIN, P.; LI., XUE, Q.; LI, X.; LIU, Y. Assessment of the bioactive phenolic composition of Acer truncatun seed coat as a byproduct of seed oil. Industrial Crops and Products, v. 118, p. 11-19, 2018.

FERREIRA, D. F. Sisvar: a computer statistical analysis system. Ciência e Agrotecnologia (UFLA), v. 35, n.6, p. 1039-1042, 2011.

HENRIQUE, M. A.; SILVÉRIO, H. A., NETO, W. P. F.; PASQUINI, D. Valorização de um resíduo agroindustrial, semente de manga, pela extração e caracterização de seus nanocristais de taninos que possam ser nocivos a saúde, além de diminuir o impacto ambiental, oriundo do descarte desse resíduo.

celulose. Journal of Environmental Management, Londres, v. 121, p. 202-209, 2013.

IMEH. U. e KHOKHAR. S. Distribution of Conjugated and Free Phenols in Fruits: Antioxidant Activity and Cultivar Variations. Journal of Agricultural and Food Chemistry, Washington, v. 50, p. $6.301,2002$.

MENNEN, L.; WALKER, R.; BENNETAUPELISSERO, C.; SCALBERT, A. Risk and safety of polyphenol consumption. American Journal of Clinical Nutrition, v. 81, n. 1, p. 326, 2006.

MONTEIRO, J. M.; ALBUQUERQUE, U. P.; ARAUJO, E. L.; AMORIM, E. L. C. Taninos: uma abordagem da química à ecologia. Química Nova, v. 28, p. 892-896, 2005.

NUNES, C. R.; BERNARDES, N. R.; GLORIA, L. L.; BARBOSA, J. B.; PEREIRA, S. M. F.; OLIVEIRA, D. B. Atividade antioxidante e o teor de taninos e fenóis totais de frutos de Annona muricata L. Vértices, Campos dos Goytacazes/RJ, v.15, n. 3, p. 93-110, 2013.

OLIVEIRA, D.S., AQUINO, P. P., RIBEIRO, S.M.R.; PROENÇA, R. P. C.; SANT'ANA, H. M. P. Vitamina $C$, carotenoide, fenólicos totais e atividade antioxidante de goiaba, manga e mamão procedentes da Ceasa do Estado de Minas Gerais. Acta Scientiarum. Health Sicences.V. 33, n. 1, p. 89-98, 2011.

ROCHA,W. S., LOPES R. M., SILVA, D. B., VIEIRA, R. F., SILVA, J. P., AGOSTINI-COSTA, T. S. Compostos fenólicos totais e taninos condensados em frutas nativas do cerrado. Revista Brasileira de Fruticultura, v.33, n.4, p 1215-1221, 2011.

SALUNKHE, D. K.; CHAVAN, J. K.; KADAM, S. $S$. Dietary tannins: consequences and remedies. Boca Raton: CRC Press, 200p, 1990. 
SARKAR, S. K.; HOWARTH, R. E. Specificity of vanillin test for flavanols. Journal of Agricultural and Food Chemistry, v.24, n.12, p.317-320, 1976.

SILVA, M. L. C.; COSTA, R. S.; SANTANA, A. S.; KOBLITZ, M. G. B. Phenolic compounds, carotenoids and antioxidant activity in plant products. Seminário: Ciências Agrárias, v. 31, n. 3, p. 669-682, 2010.

SILVA, M. R. e SILVA, M. A. A. M. P. Aspectos nutricionais de fitatos e taninos. Revista de Nutrição. v.12, n. 1, p. 21-32, 1999.

VIEIRA, G. H. F.; MOURÃO, J. A.; ÂNGELO, A. M.; COSTA, R. A.; VIEIRA, R. H. S. F. Antibacterial effect (in vitro) of Moringa oleifera and Annona muricata against gram positive and gram negative bactéria. Rev. Inst. Med. Trop, São Paulo, v. 52, n. 3, p. 129-132, 2010. 